\title{
Posterior nasal neurectomy in Hiroshima
}

\author{
Kazunori Kubota, Sachio Takeno, Takayuki Taruya, Atsushi Sasaki, Takashi Ishino, Katsuhiro Hirakawa
}

Department of Otorhinolaryngology, Head and Neck Surgery, Hiroshima University

Sensory irritation to nasal mucosa is perceived by inferior turbinate sensory nerves derived from the trigeminal nerve. The stimulation of nasal mucosa is reflected by organs such as the superior salivary nucleus via the central nerve ganglion. There are parasympathetic preganglionic cells in this ganglion, and they send parasympathetic preganglionic nerve fibers to the pterygopalatine ganglion and submandibular ganglion on the same side. In severe perennial allergic rhinitis patients, the thresholds of nerve reflection are greatly decreased, resulting in sneezing and itchiness. Rhinorrhea symptoms result from exacerbated nasal secretions due to the excitement of the cholinergic efferent parasympathetic nerve. Posterior nasal neurectomy (PNN) is a surgery invented in Japan. A PNN improves the nasal symptoms of allergic rhinitis patients by identifying the sphenopalatine artery in the posterior part of the nasal cavity and amputating the afferent and efferent nerve fibers accompanying the artery.

We demonstrated previously that the PNN procedure can lead to an inhibited orchestration of allergic inflammatory responses. Histological examinations showed that the number of inflammatory cells is markedly reduced in the lamina propria, and the empty space they leave is filled by collagen fibers. The epithelial layer is covered with stratified columnar cells. The changes in cytokine levels in nasal lavage after PNN were remarkable. The levels of interleukin (IL)-5 and eotaxin were significantly decreased after PNN (Ogawa et al. ANL 2007).

Here we provide the surgical protocol for a PNN as performed at our department, and we report the effectiveness of PNN performed for severe allergic rhinitis patients at our department within the past 3 years following our analysis of the changes in symptoms and drug scores from before to after the surgery and the changes in the nitric oxide concentration in the nasal cavity. 


\title{
Recent advances in management of allergic rhinitis in Egypt
}

\author{
M A Ragaee, A Anter, A H Monib, M M Osman, A Abdelaleem, M M Ragab
}

Department of Otorhinolaryngology, Assiut University, Faculty of Medicine, Assiut, Egypt

Affiliated to Department of Otorhinolaryngology, Hiroshima University, School of Medicine, Hiroshima, Japan

Respiratory tract allergies are known diseases from ancient Egyptian ages as delineated in the Ebers Papyrus (c. 1550 BC), which contained an impressive number of remedies for maladies including asthma.

Allergic rhinitis has been rising throughout the late $20^{\text {th }}$ and $21^{\text {th }}$ centuries and it is more obvious in Egypt due to over crowdedness and low socioeconomic level for most of the Egyptian population. Usually patients come with complications as chronic rhinosinusitis, sleep disorders and lower respiratory tract infection.

The treatment modalities became widespread including medical and surgical ways. Medical treatments includes topical corticosteroids, nasal douches, oral corticosteroids, antihistamines and mast cell stabilizers and to lesser extent immunotherapy.

Surgical intervention is limited to cases of hypertrophied inferior turbinate, complicated with chronic rhinosinusitis and nasal polyposis.

In this session, I will present the recent topics and some recent methods used in management of allergic rhinitis in the last 5 years in Egypt either introduction of new medicines or new surgical techniques. 


\title{
The Epidemiology of Chronic Rhinosinusitis and Allergic Rhinitis in Indonesia
}

\author{
Abdul Kadir \\ Dr. Wahidin Sudirohusodo Hospital Makassar Makassar
}

Background: Epidemiological and prevalence data for chronic rhinosinusitis (CRS) and allergic rhinitis (AR) in Indonesia — the fourth-most-populous country, with an estimated population of over 252 million people - are relatively rare. Purpose and settings: Our initial survey was aimed to measure the prevalence of chronic rhinosinusitis and allergic rhinitis among the patient visiting secondary to tertiary ENT care unit (or University Hospitals and it's satellites clinic). Our data has been collected secondary from medical records during 2009 until 2014. Result: On epidemiologic grounds, chronic nasal complaints represent about $50 \%$ of the total number of outpatient visits to an average Indonesian ENT practice, $18 \%$ of them have some typical symptoms of allergic rhinitis. Some association has been found between CRS prevalence and air pollution (include occupational background), active cigarette smoking, secondhand smoke exposure, allergic rhinitis, and gastroesophageal reflux. Rhinosinusitis is still the most common diagnosis for which an antibiotic is prescribed. Keyword : Rhinosinusitis, Allergic Rhinitis, Epidemiology, Indonesia 